\title{
Mathematical Programming Approach for the Design of Intensified Thermally Coupled Distillation Sequences.
}

\author{
José A. Caballero*, Juan A. Reyes-Labarta. \\ Institute of Chemical Process Engineering, University of Alicante. BO 99, 03080, Spain. \\ caballer@ua.es
}

\begin{abstract}
In this work we present a methodology that transform insights from the conceptual design of intensified thermally coupled distillation in a set of logical expressions and mathematical models that can be integrated in a mathematical programming environment with the objective of systematically generate column sequences with a reduced number of actual columns (lower than $\mathrm{N}-1$ columns, where $\mathrm{N}$ is the number of components). Some of the alternatives considered are Divided Wall Columns (DWC); Columns with vertical partitions, Kaibel like configurations, Elimination of transfer blocks, Extended DWC (including columns with vertical partitions) with external liquid transfer.

The combination of all these alternatives provides a new rich space of alternatives, some of them not known before that should be taken into account in the design of distillation based separation systems. Some examples illustrate the procedure.
\end{abstract}

Keywords: Thermally Coupled Distillation, MINLP, Intensification, Divided Wall Column, Columns with Vertical Partitions.

\section{Introduction}

Thermally Coupled Distillation (TCD) has acquire interest because the reduction in energy consumption when compared to conventional distillation systems. The reduction range from $10 \%$ to more than 50\% with typical values around $20-30 \%$.

Assuming that the energy consumption is the dominant cost, and based on structural considerations Agrawal, (2003) established that search space must be initially constrained to the set of «basic sequences», these results were corroborated by different researches, see for example Giridhar \& Agrawal, (2010). A basic sequence is a set of separation tasks that can be arranged in $\mathrm{N}-1$ actual columns (being $\mathrm{N}$ the number of key components to be separated).

Configurations with more than N-1 columns (usually called non-basic) have always higher operating costs than the best basic configuration (Caballero \& Grossmann, (2006)). Besides, non-basic configurations tend to have higher capital cost due to the extra distillation columns, and therefore non-basic configurations can be removed from the search space. Sequences with less than N-1 columns (Intensified Sequences hereinafter) have also higher operating costs than the best basic configuration, however this penalty is not too large in many situations and the reduced number of columns could eventually compensate the extra energy consumption. In the literature some of 
these cases can be found, for example those due to Brugma, (1942), Kaibel, (1987), Kim \& Wankat, (2004) or Errico et al., (2009).

These alternatives can be generated from some basic configurations by removing or merging column sections in a single shell. From a theoretically point of view it would be possible to take into account all these alternatives from the very beginning in the design of the distillation sequence, but this is not a practical approach because most of them can be generated from the set of thermodynamically equivalent configurations (TEC) the total number of alternatives considering TEC is around $2 \cdot 10^{5}$. (Caballero \& Grossmann, (2003)). All TECs have the same operating cost and usually very similar capital cost, therefore the decision on which final configuration is selected must be based on other considerations like operability and controllability. Instead, it is convenient start only with sequences of separation task, explicitly excluding non-basic sequences, but maintaining all the alternatives that could be eventually rearranged in $\mathrm{N}$ 1 columns or less. Generation of Intensified alternatives for a sequence of separation tasks.

The generation of logical relationships using Boolean or binary variables that can be included in a mathematical programming model, and that systematically include all the sequence of separation task that can be rearranged in N-1 distillation columns, was developed by Caballero and Grossmann in a set of papers -See for example, Caballero \& Grossmann, (2014)-. As this model formulation does not rely on any specific column configuration, it is considered here as the starting point for generating intensified sequences.

For the generation of intensified configurations we consider the following situations:

Divided wall columns (DWC). Basically, three distillation columns integrated in a single shell divided by a wall. (The wall is not 'touching' the upper of the lower side of the column). A DWC separates three key components in a single shell. Columns with Vertical Partitions (CVP) are similar to the DWC, but the wall is touching the upper or lower side of the shell. CVP integrates two columns in a single shell.

If we neglect the heat transfer effects through the dividing wall, DWCs and CVPs can be considered thermodynamically equivalent configurations to the non-integrated sequence. Therefore, we only need to identify which conditions allow to generate a DWC or a CVPs. Starting with any configuration among all the thermodynamically equivalent alternatives the following rules allow generating CVPs and DWC:

a.- Two columns connected by just a single thermal couple can be rearranged in a CVP. (See Figure 1a).

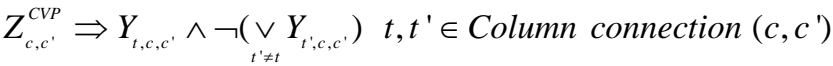

Where $Y_{t, c, c^{\prime}}$ is a Boolean variable that takes the value "True" if there is a thermal couple between columns $c$ and $c^{\prime}$ ' and $Z_{c, c^{\prime}}^{C P V}$ is a Boolean variable that indicates that columns c and c' can be merged in a single shell with a vertical partition.

b.- Two columns connected by a thermal coupled and a regular heat exchanger, can also be rearranged in a CVP, but in this case the stream connecting both columns must be externally transferred to the correct side of the column. Figure $1 \mathrm{~b}$ clarify this point. 


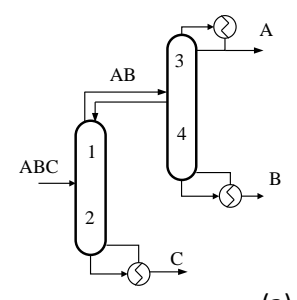

(a)

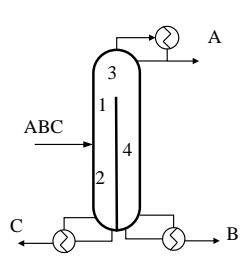

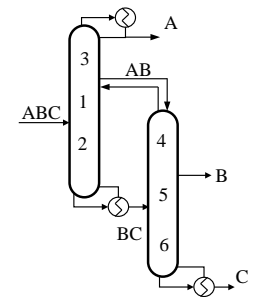

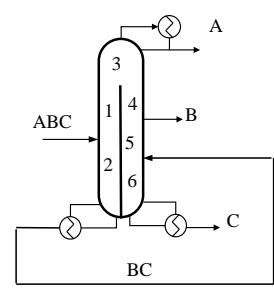

(b)

Figure 1. (a) Arrangement of two distillation columns connected by a single thermal coupled in a Column with a Vertical Partition. (b) Rearrangement in a single shell of two column connected by a thermal couple and a regular heat exchanger. Note that stream BC must be transferred to the correct position in the right side of the wall.

c.- Two columns connected by two thermal couples, without any connection between them, can be rearranged in a DWC.

$Z_{c, c^{\prime}}^{D W C} \Rightarrow Y_{t, c, c^{\prime}} \wedge Y_{t^{\prime}, c, c^{\prime}} \wedge \neg Y_{t^{\prime \prime}, c, c^{\prime}}\left\{\begin{array}{l}t, t^{\prime}, t^{\prime \prime} \in \text { Column connection }\left(c, c^{\prime}\right) \\ t<t^{\prime \prime}<t^{\prime}\end{array}\right.$

Where $Z_{c, c^{\prime}}^{D W C}$ is a Boolean variable that indicates that columns c and c' can be merged in a single shell. Note that while a CVP integrate two separation tasks in a single shell, a DWC integrates three separation task. An alternative approach for generating DWCs directly from the sequence of separation tasks can be found in Caballero \& Grossmann, (2013).

d.- If two columns are connected by more than two streams then we must differentiate two cases. 1 . The intermediate connections are liquid streams, and 2. The intermediate connection is a thermal couple. In the first case, again we can transfer the liquid streams directly to the correct part of the wall (See Figure 2.a). In the second case it is possible allowing a bidirectional transfer flow through the wall (In fact we have two nonoverlapping walls) See Figure 2.b. However, in this case there are difficulties in the operation of the column. The external transfer of the thermal couple, although possible, is not practical because a compressor is required for the vapor stream. Fortunately, Rong \& Turunen, (2006) have shown that in most situations it is possible to substitute this internal thermal couple by a liquid stream without important energy penalties. Following this approach we can transform case 2 configurations to case 1 configurations. 

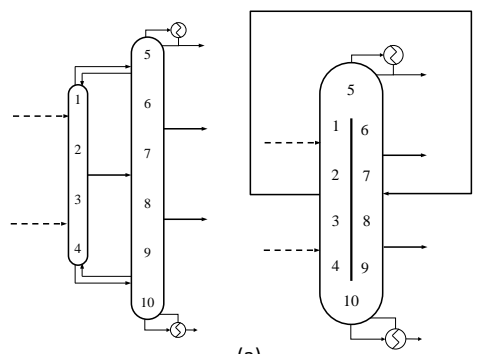
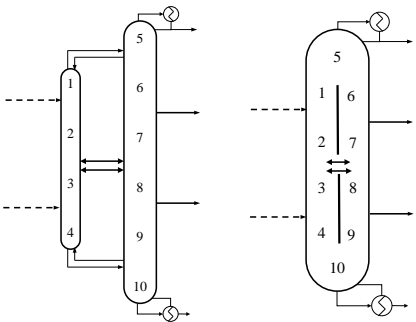

(b)

Figure 2. (a) Example of how to generate a DWC by transfer the liquid stream connecting the two columns. (b) DWC with two vertical walls and liquid and vapor transfer in the intermediate zone.

e.- Kaibel like configurations. Kaibel, (1987) introduced a DWC that separates four components using a single shell. The energy consumption of a Kaibel column is always larger than the best basic sequence but reducing from 3 two just one column have an important effect in the capital cost. Kaibel columns can be included in any sequence with more than 4components. For example, in a 5 component mixture it is possible to identify 14 sequences using 2 columns in which one of those is a Kaibel column. (Figure 3, shows as an example 4 of those alternatives).
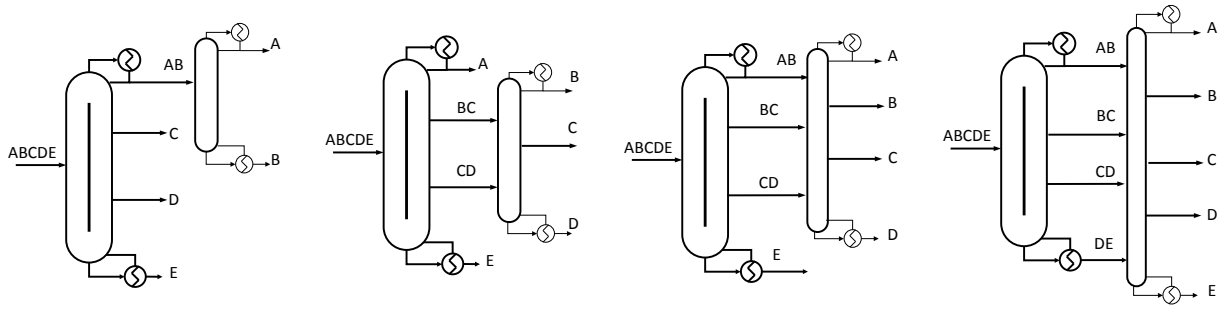

Figure 3. 4 out of the 14 possible alternatives of separating a 5 component mixture with two columns using one Kaibel column.

Removing column sections (transfer blocks). Errico and coworkers - see for example Errico et al., (2014)- showed that it is possible to remove some intermediate columns formed by a single section without excessive energy penalty and therefore reducing the number of actual columns (See Figure 4.a-c). For a given sequence of separation tasks, the sequences that include at least a column with a single section, if any, appear as a subset of the thermodynamically equivalent (TE) alternatives. Caballero \& Grossmann, (2003) proposed a set of logical relationships for obtaining all the TE for a given sequence of tasks. Using that set of equations and enforcing that at least one column is formed by a single section allows easily to obtain those configurations. 


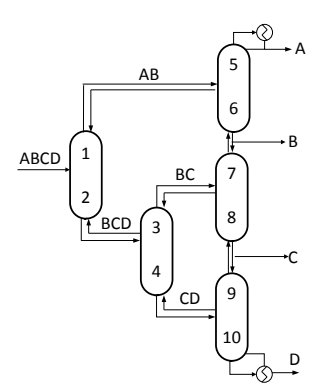

(a)

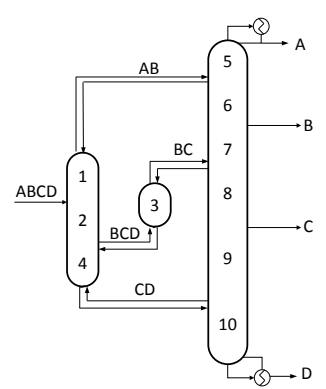

(b)

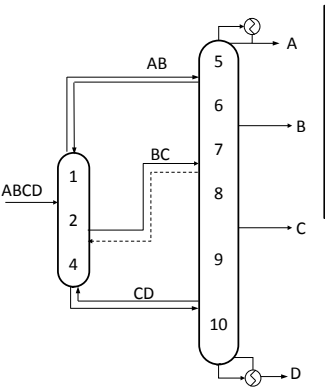

(c)

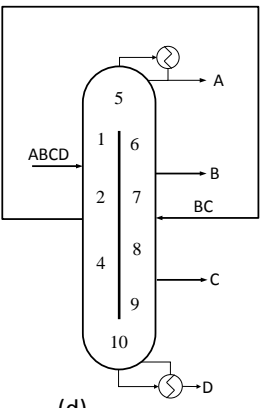

(d)

Figure 4. (a-c) Steps to remove a transfer block. (a) Sequence of separations, each column is a separation task. (b) Rearrangement in three columns with a column formed by a single section. (c) Sequence after removing section 3. (d) Final arrangement in a single column with an internal wall and external liquid transfer.

It is worth remarking that removing a transfer block is equivalent to withdraw an intermediate stream from a column. If we try to withdraw a pure product or a stream that completely separate a given component the energy requirements can be huge and/or there is an upper bound in the purity (recovery). However, in this case the components extracted are distributed along the column (Figure 4.c)

After removing some 'transfer blocks' it could be possible to reduce even more the number of columns by searching in the resulting sequence new DWCs or CVP -Figure 4.d).

\section{Example}

Consider the separation of a mixture of 5 linear hydrocarbons, from n-C5 to n-C10. With the data provided in Table 1 . The optimal solution considering only basic sequences is a sequence with a large degree of thermal couple, but with two condensers and two reboilers (see Figure 5a). The internal couples associated to internal mixtures (BCD, BC and CD) can be substituted by a single liquid stream with almost no penalty in energy (or cost) less than $1 \%$. For this configuration there are no transfer blocks, however there are different thermodynamically equivalent alternatives for generating intensified sequences involving three or two columns. The Figure $5 \mathrm{~b}$ shows a feasible configuration using two actual columns with external transferences liquid streams with a TAC reduction around $10 \%$

Table 1. Data for the example.(Rest of physical data from Aspen-Hysys ${ }^{\text {TM }}$ database)

\begin{tabular}{lc|ll}
\hline Components & $\begin{array}{c}\text { Feed flow } \\
(\mathrm{kmol} / \mathrm{h})\end{array}$ & & \\
\cline { 1 - 2 } $\mathrm{A}=$ n-hexane & 120 & Pressure & $1.0 \mathrm{~atm}$ \\
$\mathrm{~B}=$ n-heptane & 90 & Feed liquid fraction & 1 \\
$\mathrm{C}=$ n-octane & 60 & Vapour Steam cost & $14.05 \$ / \mathrm{GJ}$ \\
$\mathrm{D}=$ n-nonane & 30 & Cooling water cost & $0.354 \$ / \mathrm{GJ}$ \\
$\mathrm{E}=$ n-decane & & Cost data source & Turton et al (2013) \\
\hline
\end{tabular}



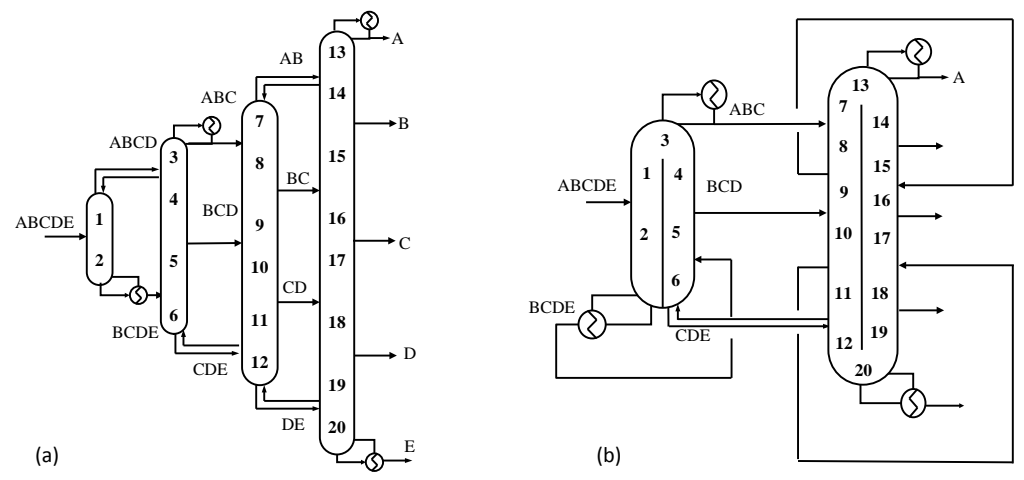

Figure 5. (a) A feasible arrangement in $\mathrm{N}-1$ columns for the example optimal solution. (b) A feasible arrangement in two columns. The configuration includes a CVP and a DWC with external liquid transfer.

\section{Conclusions}

A systematic procedure for the synthesis and intensification of column configurations for multicomponent distillation is presented. The algorithm starts by searching in the space of basic configurations extended with the internal structure of heat exchangers. It has been shown that some internal heat exchangers can have a large impact on the performance of the complete system. On the other side it is not convenient to explicitly consider, at this stage, the final arrangement in actual columns. This is because there are a potential very large number of thermodynamically equivalent configurations with similar cost.

Taking as a starting point a basic sequence it is possible systematically generate intensified sequences by searching for DWCs, CVPs. It the heat transfer across the wall is not affecting the column operation, all these configurations are thermodynamically equivalent (no energy penalty). If it is possible to substitute internal thermal couples by liquid streams without important energy penalties, then we can generate a new set of DWCs or CVPs by externally transfer these liquid streams to the correct side of the wall. Searching in the space of thermodynamically equivalent configurations, some configurations present columns with a single section (transfer blocks) that in most situations can be removed without too much energy penalty, reducing by one the number of actual columns. When a transfer block is removed, then new opportunities for generating new DWC/CVPs appear. The example shows that it is possible reduce until just two columns with internal walls the sharp separation of a 5 component mixture that usually requires 4 columns.

\section{Acknowledgements.}

The authors wish to acknowledge the financial support by the Ministry of Economy and Competitiveness of Spain and to the EU, under the project CTQ2012-37039-C02-02.

\section{References}

Agrawal, R. 2003. Synthesis of multicomponent distillation column configurations. AIChE Journal, 49, 379-401.

Brugma, A. J. 1942. Process and Device for Fractional Distillation of Liquid Mixtures. U.S. Patent 2.295.256. 
Caballero, J. A., \& Grossmann, I. E. 2003. Thermodynamically Equivalent Configurations for Thermally Coupled Distillation. Aiche Journal, 49, 2864-2884.

Caballero, J. A., \& Grossmann, I. E. 2006. Structural considerations and modeling in the synthesis of heat-integrated-thermally coupled distillation sequences. Industrial and Engineering Chemistry Research, 45, 8454-8474.

Caballero, J. A., \& Grossmann, I. E. 2013. Synthesis of complex thermally coupled distillation systems including divided wall columns. Aiche Journal, 59, 1139-1159.

Caballero, J. A., \& Grossmann, I. E. (2014). Optimization of Distillation Processes. In A. Górak \& E. Sorensen (Eds.), Distillation Fundamentals and Principles. . London: Elsevier.

Errico, M., Pirellas, P., Torres-Ortega, C. E., Rong, B.-G., \& Segovia-Hernandez, J. G. 2014. A combined method for the design and optimization of intensified distillation systems. Chemical Engineering and Processing: Process Intensification, 85, 69-76.

Errico, M., Rong, B.-G., Tola, G., \& Turunen, I. 2009. A method for systematic synthesis of multicomponent distillation systems with less than N-1 columns. Chemical Engineering and Processing: Process Intensification, 48, 907-920.

Giridhar, A., \& Agrawal, R. 2010. Synthesis of distillation configurations: I. Characteristics of a good search space. Computers \& Chemical Engineering, 34, 73.

Kaibel, G. 1987. Distillation Columns with Vertical Partitions. Chemical Engineering Techology, 10, 92-98.

Kim, J. K., \& Wankat, P. C. 2004. Quaternary distillation systems with less than N-1 columns. Industrial \& Engineering Chemistry Research, 43, 3838-3846.

Rong, B. G., \& Turunen, I. 2006. New heat-integrated distillation configurations for Petlyuk arrangements. Chemical Engineering Research \& Design, 84, 1117-1133. 